\title{
Current Computational Models for Prediction of the Varied Interactions Related to Noncoding RNAs
}

\author{
Xing Chen, ${ }^{1}$ Huiming Peng, ${ }^{2}$ and Zheng Yin ${ }^{3}$ \\ ${ }^{1}$ School of Information and Electrical Engineering, China University of Mining and Technology, Xuzhou 221116, China \\ ${ }^{2}$ Department of Math, Science \& Technologies, Forsyth Technical Community College, Winston-Salem, NC, USA \\ ${ }^{3}$ Department of Systems Medicine and Bioengineering, Houston Methodist Research Institute, Houston, TX, USA \\ Correspondence should be addressed to Xing Chen; xingchen@amss.ac.cn
}

Received 19 October 2016; Accepted 20 October 2016

Copyright (C) 2016 Xing Chen et al. This is an open access article distributed under the Creative Commons Attribution License, which permits unrestricted use, distribution, and reproduction in any medium, provided the original work is properly cited.

Noncoding RNA (ncRNA) refers to a kind of endogenous small RNA molecules that have no protein coding capacity. In recent years, extensive studies have been conducted to study the roles of ncRNAs in cell biology, and accumulating evidences show that these RNA molecules do not constitute transcriptional noise but play important roles in cellular functions, such as transcriptional and posttranscriptional regulation, epigenetic regulation, organ or tissue development, cell differentiation, cell cycle control, cellular transport, metabolic processes, and chromosome dynamics. Their deregulation heavily contributes to various pathological conditions of human complex diseases, including breast cancer, hepatocellular cancer, prostate cancer, colon cancer, bladder cancer, thyroid cancer, lung cancer, ovarian cancer, leukemia, Alzheimer's disease, diabetes, and HIV. The development of computational models to predict the various complex ncRNA-related interactions could significantly benefit the inference of ncRNA function, the identification of ncRNAdisease associations, the detection of ncRNA biomarker, the identification of drug-target interactions, and drug design.

The papers that follow in the next pages describe recent findings in the field of computational models for prediction of the varied interactions related to ncRNAs. They represent only a fraction of the current research, the emerging trends, and applications of computational models for ncRNAs. The special issue consists of eight research papers and one review paper. The research papers include identification and annotation of ncRNAs (four papers), miRNA-target interaction prediction (one paper), ncRNA function prediction (one paper), miRNA-transcriptional factor interaction prediction (one paper), and protein-protein interaction prediction (one paper). A brief description of the papers follows.

G. Wan et al. analyzed RNA-seq data and ChIPseq histone modification data to identify the relationship between lncRNA genes transcription and histone modification $\mathrm{H} 3 \mathrm{~K} 4 \mathrm{me} 3$ or H3K27me3 in the paper "Transcriptional Regulation of lncRNA Genes by Histone Modification in Alzheimer's Disease."

J. Qu et al. detected 254 small noncoding RNAs in genome of Pleurotus ostreatus and analyzed the evolutionary conservation of them in the paper "Identification and Characterization of Small Noncoding RNAs in Genome Sequences of the Edible Fungus Pleurotus ostreatus."

A meta-path-based prediction method RMLM was developed in the paper "A Meta-Path-Based Prediction Method for Human miRNA-Target Association” by J. Luo et al. for predicting potential miRNA-target interactions. The authors also developed RMLMSe, in which sequence information was utilized to improve the performance of RMLM.

L. Jiang et al. employed backpropagation (BP) neural network together with 98-dimensional novel features for microRNA (miRNA) precursor identification in the paper "BP Neural Network Could Help Improve Pre-miRNA Identification in Various Species." The authors demonstrated that the total prediction accuracy of this method was nearly $13.17 \%$ greater than the state-of-the-art miRNA precursor prediction software tools. 
A framework named Gene2Function to annotate Gene Reference into Functions (GeneRIFs) was given by Y. Hu et al. in the paper "Annotating the Function of the Human Genome with Gene Ontology and Disease Ontology."

M-fold, TargetScan, and GeneCoDia3 were used in the paper "Human Ribosomal RNA-Derived Resident MicroRNAs as the Transmitter of Information upon the Cytoplasmic Cancer Stress" for investigating RNA relationships between rRNA and miRNA against cellular stresses by M. Yoshikawa and Y. R. Fujii. The authors detected 17 RNA sequences identical with known miRNAs in the human rRNA and termed as rRNA-hosted miRNA analogs (rmiRNAs).

The paper "Ens-PPI: A Novel Ensemble Classifier for Predicting the Interactions of Proteins Using Autocovariance Transformation from PSSM" by Z.-G. Gao et al. provided a novel predictor based on the Rotation Forest (RF) algorithm combined with Autocovariance (AC) features extracted from the Position-Specific Scoring Matrix. The method achieved promising prediction performance when implemented on the protein-protein interaction datasets of Yeast, $H$. pylori, and independent datasets.

Q. Zhao et al. constructed a computational model of miRNA-mediated feed-forward loops (FFLs) in the paper "Effect of Dynamic Interaction between MicroRNA and Transcription Factor on Gene Expression." The authors introduced four possible structural topologies of FFLs with two gate functions (AND gate and OR gate) based on the different dynamic interactions between miRNA and TF on gene expression.

Finally, in the review paper "Long Noncoding RNA Identification: Comparing Machine Learning Based Tools for Long Noncoding Transcripts Discrimination" by S. Han et al., several popular methods for lncRNA identification such as Coding Potential Calculator (CPC), Coding-Potential Assessment Tool (CPAT), Coding-Non-Coding Index (CNCI), predictor of long noncoding RNAs and messenger RNAs based on an improved $k$-mer scheme (PLEK), Long noncoding RNA IDentification (LncRNA-ID), IncRScan-SVM, lncRNA-MFDL, and LncRNApred were summarized with their advantages, disadvantages, and application scopes.

\section{Acknowledgments}

We would like to thank the authors who submitted their work for consideration to this special issue as well as the reviewers for their efforts and constructive criticism. The work of Xing Chen was supported by National Natural Science Foundation of China under Grant nos. 11301517 and 11631014.

Xing Chen

Huiming Peng

Zheng Yin 

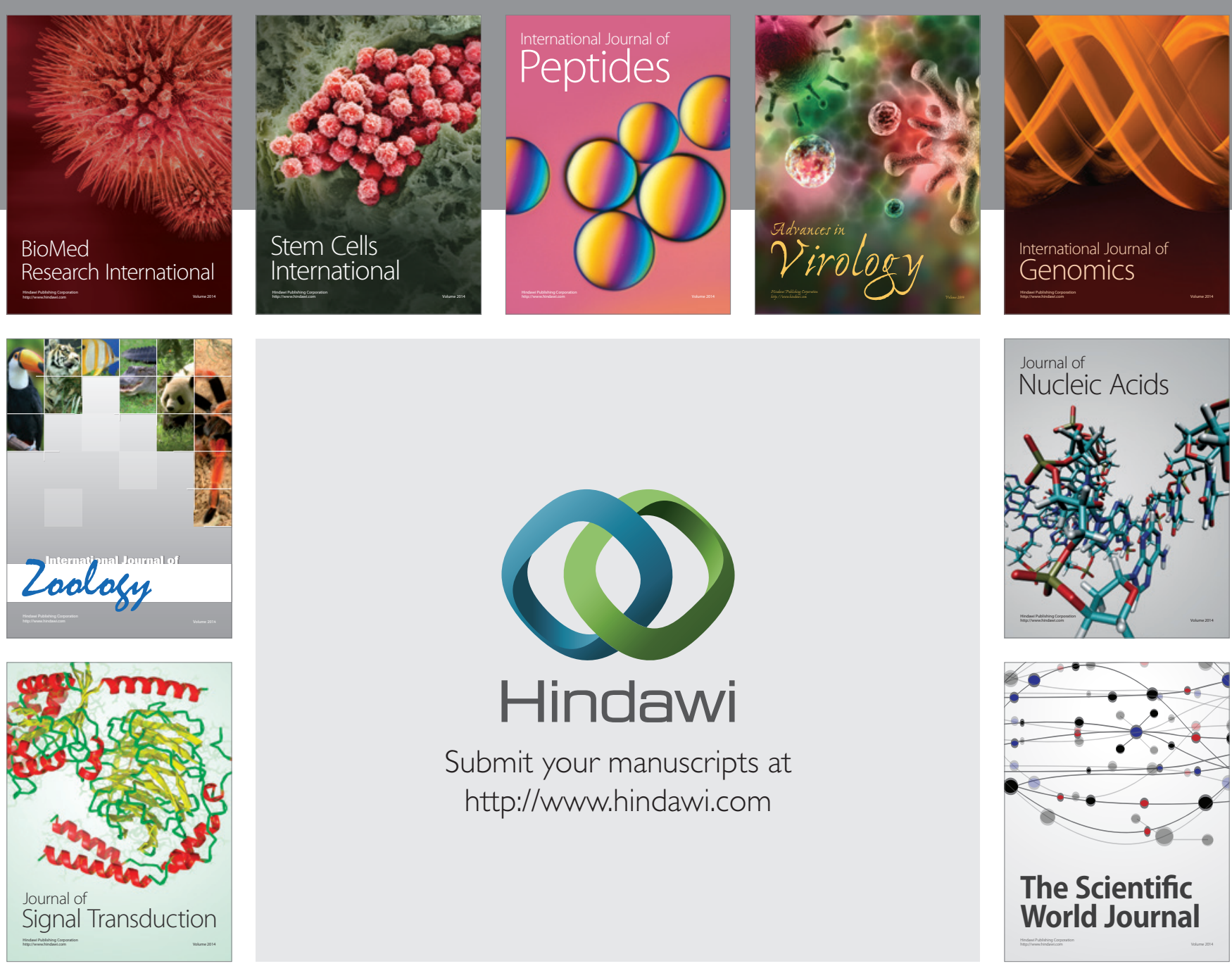

Submit your manuscripts at

http://www.hindawi.com
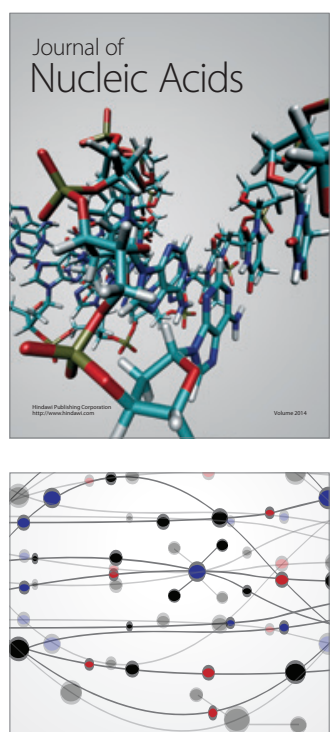

The Scientific World Journal
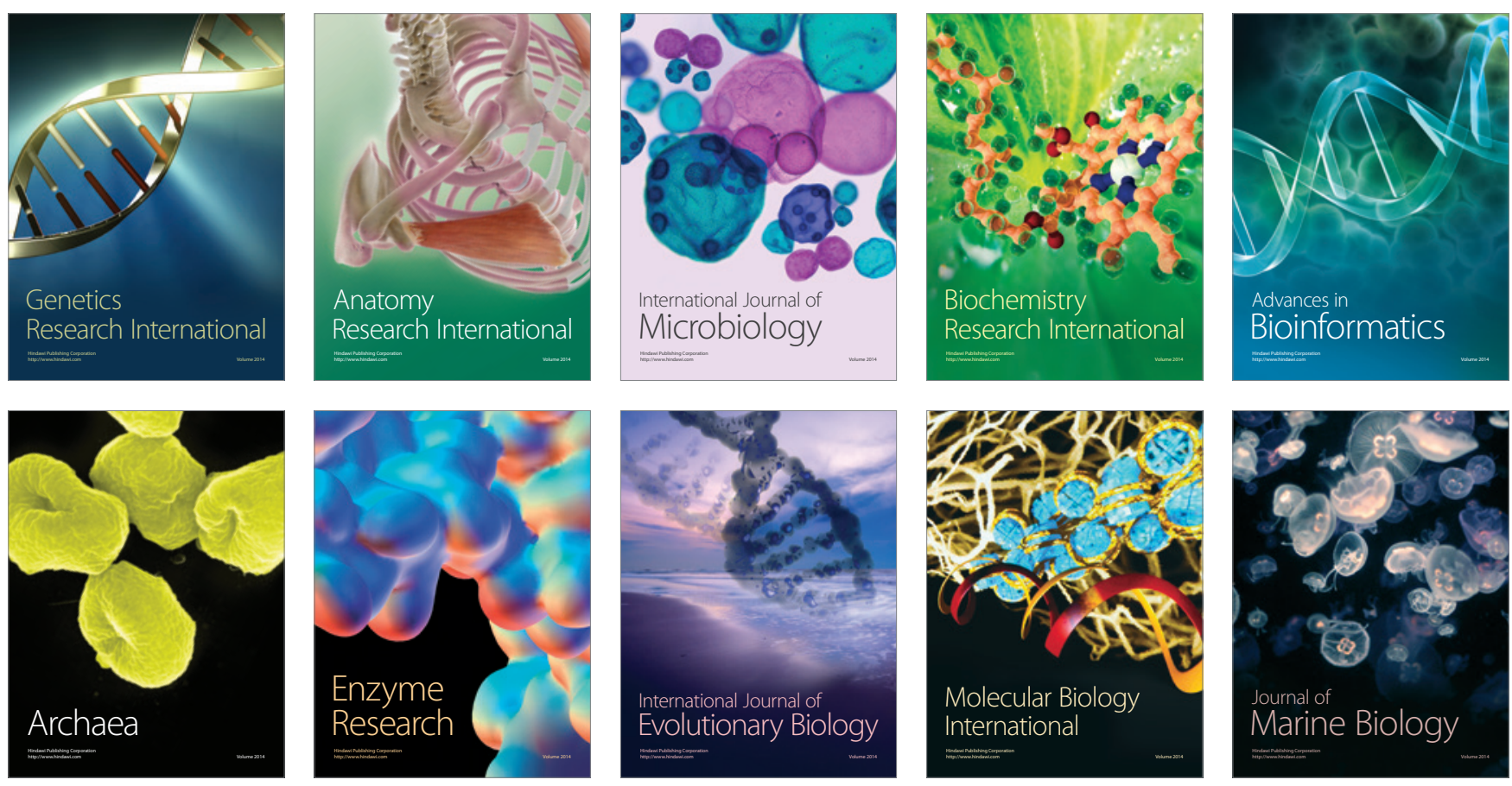